\title{
Title: Technology-Driven Layer-by-Layer Assembly of Nanofilms
}

\author{
Authors: Joseph J. Richardson ${ }^{1}$, Mattias Björnmalm ${ }^{1}$, Frank Caruso ${ }^{1}$ *
}

\begin{abstract}
Affiliations:
${ }^{1}$ ARC Centre of Excellence in Convergent Bio-Nano Science and Technology, and the Department of Chemical and Biomolecular Engineering, The University of Melbourne, Parkville, Victoria 3010, Australia.

*Correspondence to: E-mail: $\underline{\text { fcaruso@unimelb.edu.au }}$
\end{abstract}

\begin{abstract}
Multilayer thin films have garnered intense scientific interest due to their potential application in diverse fields such as catalysis, optics, energy, membranes and biomedicine. Here, we review the current technologies for multilayer thin film deposition using layer-by-layer assembly, and discuss the different properties and applications arising from the technologies. We highlight five distinct routes of assembly, each of which offers unique material and processing advantages for assembling layer-by-layer films: immersive, spin, spray, electromagnetic, and fluidic assembly. Each technology encompasses numerous innovations for automating and improving layering, which is important for research and industrial applications. Furthermore, we discuss how judicious choice of the assembly technology enables the engineering of thin films with tailor-made physicochemical properties, such as distinct layer stratification, controlled roughness, and highly ordered packing.
\end{abstract}




\section{Structured abstract / one page summary:}

Background: Over the last few decades, the layer-by-layer (LbL) assembly of thin films has been of considerable interest because of its ability to exert nanometer control over film thickness and its extensive choice of usable materials for coating planar and particulate substrates. The choice of materials allows for responsive and functional thin films to be assembled with engineered film properties for various applications, including catalysis, optics, energy, membranes, and biomedicine. Furthermore, there is now a growing realization that the assembly methods and technologies significantly impact the physicochemical properties, and ultimately the performance of the thin films.

Advances: Recent advances in LbL assembly technologies have explored novel driving forces for the assembly process when compared with the diffusion-driven kinetics of classical LbL assembly, where a substrate is immersed in a polymer solution. Examples of different assembly technologies that are now available include: dipping, dewetting, roll-to-roll, centrifugation, creaming, calculated-saturation, immobilization, spinning, high gravity, spraying, atomization, electrodeposition, magnetic assembly, electrocoupling, filtration, microfluidics, and fluidized beds. These technologies can be condensed into five broad categories where automation or robotics can also been applied, namely (i) immersive, (ii) spin, (iii) spray, (iv) electromagnetic, and (v) fluidic assembly. Many of these technologies are still new and are actively being explored, with research shedding light on how the deposition technologies and the underlying driving forces impact the formation, properties and performance of the films, as well as the ease, yield and scale of the processing.

Outlook: LbL assembly has proven remarkably powerful over the last two decades, and has had a profound interdisciplinary impact on scientific research. Scaling up the process is crucial for furthering real world applications, and moving forward, an understanding of how to carefully select assembly methods to harness the specific strengths of different technologies has the potential to be transformative. Comprehensive comparisons between the technologies still need to be conducted, especially in regards to coating particulate substrates, where comparisons are limited but crucial for advancing fundamental research and practical applications. 
Summary image caption

The layer-by-layer assembly of nanofilms for preparing functional materials. The properties and performance of the resulting films depend on the substrate and layer choices, as well as the assembly technology.

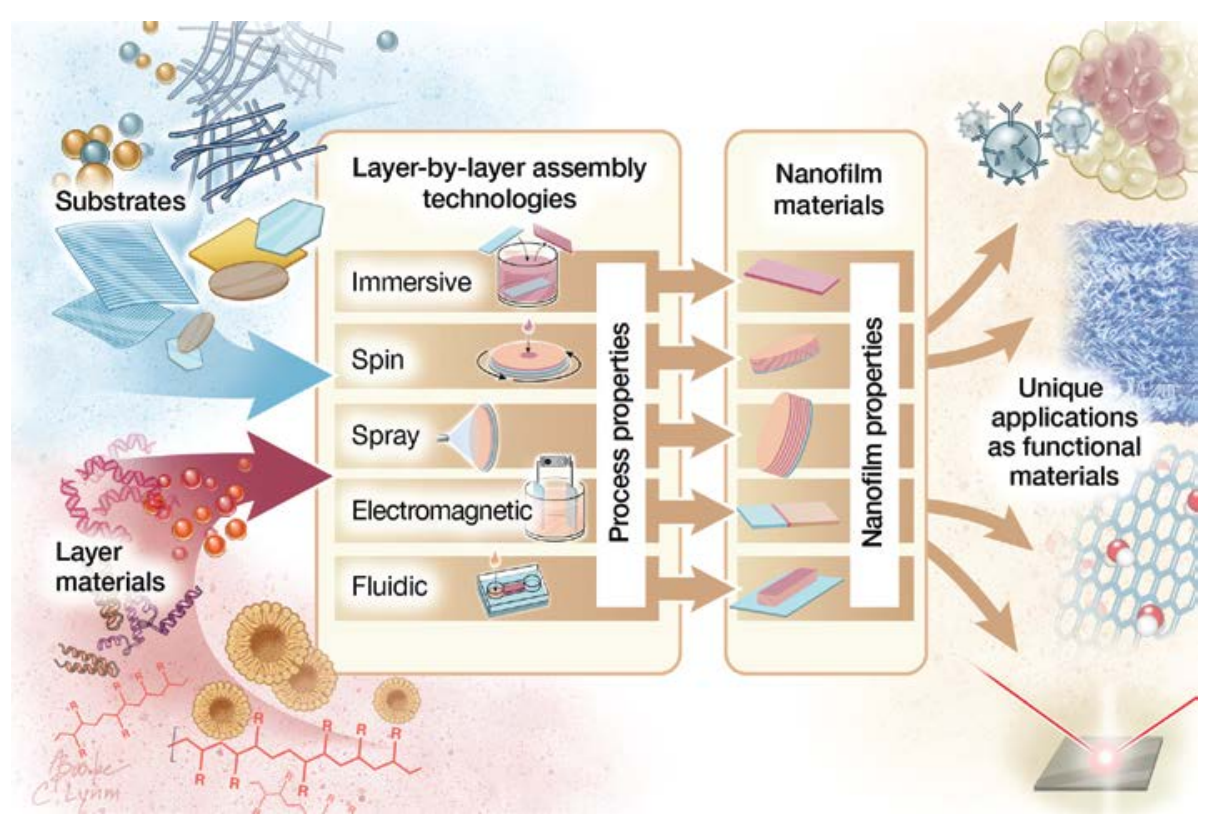


Main Text: The performance of functional materials is governed by their ability to interact with surrounding environments in a well-defined and controlled manner. Whether harnessing photons or electrons, separating out gas molecules or solutes, or responding to biomolecules or organisms, the environment-material interface is essential in determining the performance of the materials in various applications. Coating technologies provide the means to control the surface of a material, thus creating composite materials where the interface and the bulk of the material can, to a large extent, be engineered and controlled independently.

Layer-by-layer (LbL) assembly is a prevalent method for coating substrates with functional thin films. Following early studies that report multilayer assembly $(1,2)$, it is only in the past two decades that the field has witnessed considerable growth (3). Generally, LbL assembly is a cyclical process where a charged material is adsorbed onto a substrate, and after washing, an oppositely charged material is adsorbed on top of the first layer. This constitutes a single bilayer with a thickness generally on the order of nanometers, and the deposition process can then be repeated until a multilayer film of desired thickness has been assembled (3). For certain applications the substrate can then be removed, yielding freestanding macroscopic films such as membranes (4), or freestanding micro- or nanoscopic films such as hollow capsules (5, 6). Although electrostatic interactions remain widely used in facilitating formation of the films, other molecular interactions (e.g., covalent, hydrogen-bonding, host-guest) are now well established for LbL assembly, with diverse materials (e.g., polymers, proteins, lipids, nucleic acids, nanoparticles, suprastructures) used as film constituents (7). The simplicity, versatility and nanoscale control that LbL assembly provides makes it one of the most widely used technologies for coating both planar and particulate substrates in a diverse range of fields, including optics, energy, catalysis, separations, and biomedicine (Fig. 1A).

The widespread use of LbL assembly in fields with different standard tools and procedures, as well as the different processing requirements associated with substrates such as porous membranes, particles and biological matter, has led to the development of a number of LbL assembly technologies. Examples include: dipping (2), dewetting (8), roll-to-roll (9), centrifugation (10), creaming (11), calculated-saturation (12), immobilization (13), spinning (14), high gravity (15), spraying (16), atomization (17), electrodeposition (18), magnetic assembly (19), electrocoupling (20), filtration (21), fluidics (22), and fluidized beds (23). These different methods have largely been treated as "black boxes”, where the main focus has been on 
what materials are used (the input) for assembling the thin films (the output), with little focus placed on the actual assembly method. However, there is now a growing realization that the assembly method not only determines the process properties (such as the time, scalability and manual intervention), but also directly impacts the physicochemical properties of the films (such as the thickness, homogeneity, and inter- and intra-layer film organization), with both sets of properties linked to application-specific performance (Fig. 1B).

\section{Unpacking the "Black Box"}

The basis of LbL assembly is the sequential exposure of a substrate to the materials that will compose the multilayer films. The assembly technologies used to assemble such films form five distinct categories, namely: (i) immersive, (ii) spin, (iii) spray, (iv) electromagnetic, and (v) fluidic assembly (Fig. 2). These assembly technologies impact both the process properties and the resultant material properties (Table 1), and therefore careful choice of the assembly method can be crucial for successful application of the assembled films. Furthermore, two main themes can be identified for current developments in assembly technologies: the first is the move away from random diffusion-driven kinetics for layer deposition, and the second is the advancement from manual assembly toward automated systems.

\section{Immersive Assembly}

Immersive LbL assembly, sometimes referred to as “dip assembly”, is the most widely used method and the standard that newer technologies are often compared against. Immersive assembly is typically performed by manually immersing a planar substrate into a solution of the desired material $(2,24,25)$ followed by three washing steps to remove unbound material (26). Particulate substrates can also be layered using immersion; however, the washing and deposition steps are generally broken up by centrifugation to pellet the particles $(5,6,10)$. Early studies on using particles for depositing planar multilayers noted that theoretically any material capable of having a surface charge, such as metals, nonmetals, organics and inorganics, could be applied for growing multilayers if suitable conditions are used $(2,24,27)$. Further, it was also reported that the thickness of each layer corresponds to the thickness of the particles being adsorbed $(24,28)$. 
Immersive assembly allows for more homogenous films, both when using particle (27) or polymer multilayers (3), in comparison with non-LbL assembly technologies, such as gas deposition and nucleation deposition, making LbL assembly widely used for thin film formation.

Improvements in immersive assembly include speeding up the process by shifting the deposition kinetics away from random diffusion toward faster kinetics, such as those arising from dewetting (8), and by automating labor-intensive steps with robotic immersion machines $(24,26,29,30)$. The colloids used for planar assembly in early studies only required 1 min of immersion for each adsorption step (31); however, for immersive assembly using polymers the substrate is ideally immersed for $~ 15$ min for sufficient layer deposition $(25,26)$. To reduce the assembly time for polymers, and to allow for the deposition of low surface charge/small contact area materials, solutions doped with organic solvents (e.g., dimethylformamide) can be used to eliminate the need for rinsing and drying steps through the process of dewetting (8). Dewetting leads to a 30-fold reduction in assembly time because the adsorption process is no longer governed by diffusion, but by evaporation and dewetting. Another interesting move away from random diffusion utilizes polymer solutions that are constantly stirred by magnetic stirrer bars, which allows for robust layers to be deposited within tens of seconds after immersion (32). Instead of speeding up the adsorption process by using different adsorption kinetics, handling times can be decreased by automating the process $(9,24,26,27,29,30,33)$. One approach to automation uses a quartz crystal microbalance (QCM) as a substrate, allowing for layering to be controlled with a computer-monitored feedback loop (30). QCM enables the layering process to be based on a fixed mass of adsorbed material rather than fixed immersion times. Furthermore, the feedback loop allows for precise and reproducible control over the film growth, and allows for linear film growth to be engineered from polymer combinations that give non-linear film growth using fixed times (30). For fixed time immersive assembly, computer-programmed automated slide stainers can be retrofitted for automated multilayer assembly, allowing for agitation and solution exchange during washing steps $(24,29)$. A similar, although custom-built computer-programmed machine can deposit 1000 layers of charged colloids onto particulate substrates (substrates $\sim 100 \mu \mathrm{m}$ in diameter) (27).

Although automation decreases manual involvement, it does not significantly reduce the overall assembly time, which is why some efforts have focused on combining faster deposition kinetics with automated systems. For example, one commercially available robot uses a rotating 
slide holder to speed up the assembly process (26). This rotation allows for a three to ten-fold reduction in adsorption times and allows for thicker films to be prepared using higher rotation speeds. Roll-to-roll assembly allows for layering to be performed even quicker (5- to 10-fold) through the use of flexible substrates (9). The immersion time and speed of the rolling process play a large role in determining the film properties, and the drying conditions, wettability, and substrate movement speed required significant optimization to produce films with similar properties to standard immersive assembly (9). A further improvement on roll-to-roll assembly uses a nip-roll technique to prevent excess solution from cross-contaminating the system, resulting in more homogenous coatings than immersive assembly (34).

Immersive assembly can be performed on particulate substrates that are too small to sediment quickly or physically move between solutions, such as micro- and nanoparticles. The most common technology for immersive assembly on particulate substrates is performed by dispersing dense particulate substrates in a polymer solution, pelleting the particles with centrifugation, removing the supernatant, washing multiple times with a similar pelleting process, and then repeating the steps for multilayer growth $(5,6,10)$. This is generally time consuming and labor intensive due to the centrifugation steps, and particles dense and large enough to be pelleted are required. However, by using particulate substrates lighter than water (e.g., emulsions), creaming and skimming cycles can be applied for washing steps (11), although centrifugation can also be used to speed up the flotation and creaming process (35) with lighter emulsions capable of creaming in a matter of minutes rather than hours (36). The use of emulsions as templates results in thicker films compared with using solid templates, probably due to the surfactants used for emulsion stabilization (36).

The major driving force behind the development of novel immersive assembly technologies for particulate substrates is the attempt to avoid centrifugation, as it can lead to aggregation, is labor intensive, and is generally difficult to automate. A simple way to avoid centrifugation is to remove the need for washing steps. Avoiding washing steps can be performed by adding exact amounts of polymer calculated to saturate the surface of the particulate substrates $(12,37)$, rather than the high concentrations of excess polymer solution generally used $(5,6,10)$. Initially, only 2-3 layers could be deposited before the particles start to aggregate (12), but more layers can be deposited by incrementally measuring the zeta potential during assembly (37). Additionally, the use of constant mixing for soft particulate substrates 
such as emulsions (38) or sonication during layer deposition for hard particulate substrates like drug crystals (39) reduces aggregation. By optimizing the protocol, the saturation method gives a similar shell thickness to centrifugation-based assembly but is about three times faster $(37,39)$. This technology requires constant monitoring and surface area calculations to avoid adding excess polymer, and therefore does not reduce manual involvement. A technology that focuses on decreasing manual involvement and reducing the need for centrifugation uses particulate substrates immobilized in agarose to convert collections of particulate substrates into a macroscopic substrate (33). This macroscopic collection of immobilized particles can be treated like a planar substrate and immersed in polymer solutions using a robotic dipping machine, allowing for full automation during the layering process. Although this technology generates films roughly half the thickness of those prepared by conventional centrifugation-based assembly, likely due to the impeded diffusion of polymers through the agarose hydrogel, 80\% of the particles can be recovered, which is a significant improvement over the $\sim 90 \%$ loss that has been reported for centrifugation-based assembly at high layer numbers $(21,33)$.

Due to the ease of use and versatility of material and template choice, immersive assembly has been applied for numerous applications. For example, light-emitting diodes (LEDs) can be prepared from immersive assembly on planar substrates, with the polymer choice and multilayer thickness giving control over luminance and the turn-on voltage (29). Automated rollto-roll immersive assembly can be used for depositing conductive and flame-retardant coatings (34). Planar substrates coated with particle multilayers can be used for the detection of small particles invisible to the naked eye through color shifts in the multilayer films (2). Glass slides can also be coated with particle multilayers for the preparation of antireflective, anti-fogging, and self-cleaning surfaces (24). Fusion micro-reactors coated with particles are more conducive toward reaction (27). Certain particulate substrates easily allow for the removal of the template particle, leaving behind hollow multilayer capsules, and similarly, drugs themselves can be used as the particulate templates, with both types suitable for drug delivery, (5, 6, 33, 38-40).

In summary, immersive assembly is the most commonly used LbL assembly technology, and the de facto standard that other technologies are compared against. The simplicity of immersing substrates of almost any shape or size into containers with layering solution makes this technology easily accessible. The films produced have an interpenetrated structure and form “fuzzy nanoassemblies” that are almost synonymous with LbL assembly (3). Much recent work 
has been focused around shorter assembly times and automated systems with less manual intervention. For coating particulate substrates there has also been significant interest in technologies applicable to coating smaller, low density particles, such as silica nanoparticles, which can be difficult to handle with the conventional centrifugation-based assembly. As immersive assembly typically requires more material than other technologies, especially to submerge large substrates on industrial scales, waste can be an issue, although solutions can be reused as long as cross-contamination remains low. Immersive assembly has been the workhorse of LbL assembly and will undoubtedly continue to play an integral part in the development of new and improved thin films.

\section{Spin Assembly}

Layer-by-layer assembly using spin coating, or "spin assembly”, utilizes the common coating technology of spinning a substrate to facilitate the deposition of materials (14). Although drying a substrate after immersive LbL assembly can be achieved through spinning (41), the majority of spin assembly is performed by either casting the solution onto a spinning substrate (42), or by casting the solution onto a stationary substrate that is then spun (43). Spinning quickens the assembly process significantly, allowing for layers to be deposited in $~ 30$ seconds due to the various forces governing the process (43). Furthermore, spin assembly allows for automation and the coating of substrates up to $10 \mathrm{~cm}$ in diameter using commercially available spin coaters (44, 45). However, standard spin coaters are generally designed for flat surfaces and are not amenable to the complex shapes accessible to immersive assembly.

Spin assembly typically results in more homogenous films compared with immersive assembly. This is because assembly is driven by a collection of forces including electrostatic interactions, which cause the adsorption and rearrangement of polymers, and centrifugal, air shear and viscous forces, which cause desorption of weakly bound polymers and dehydration of the films (43). These forces are also the reason why spin assembly is orders of magnitude faster than immersive assembly. The salt concentration of the polymer solution has a larger effect at higher spin speeds, meaning that electrostatic forces play a greater role at low ionic strength, and shear forces dominate at high ionic strength (46). These shear forces produce thinner, highly ordered films with specific layer interfaces when compared with immersive assembly, which 
produces thicker interpenetrated films (47). Specifically, the thickness for spin-assembled polymer films is generally linked to the spin speed, with higher speeds leading to thinner films (42). When depositing colloids, the forces experienced during spinning lead to a monolayer of colloids, while standard immersive assembly often leads to a pseudo-monolayer where the substrate is not fully coated $(43,48)$. A comparison study of the differences between automated immersive assembly and automated spin assembly found that immersive-assembly prepared thicker, rougher films, while spinning resulted in thinner, smoother films (44). Interestingly, the films differed visually, as the spin-assembled films were transparent because of their distinct layer stratification, and the immersive-assembled films were opaque due to their inhomogeneous, interpenetrated layers (Fig. 3). The contact angle and the relative concentration of polymers was consistent across all bilayers for spin-assembled films, while immersive-assembled films became rougher with time, giving varying contact angle and relative concentration ratios between the two constituent layers (44). Another study, which compared spin assembly and immersive assembly, showed that clay nanocomposites in spin-assembled films have a higher degree of orientation (45). However, one issue that can result from spin assembly, which is not a concern for other assembly technologies, is that at higher ionic strengths of polymer solution, and also at lower spin speeds, the films can be thicker where the solution was cast when compared with the edges of the substrate $(42,46)$.

In a special case of spin assembly, the substrate can be placed in a closed container with a polymer solution or a colloidal dispersion parallel to the axis of rotation (rather than perpendicular). Upon spinning, centrifugal force pushes the layer material directly onto the substrate, rather than across the substrate, hence the name "high gravity assembly" (15). This allows for improved film deposition and uniformity, especially at low polymer concentrations, because the rotation and increased turbulence lower the thicknesses of both the laminar layer and the diffusing layer around the substrate. The adsorption equilibrium can be reached at least five times quicker than immersive assembly and is controllable by the spin speed. Furthermore, polymer combinations that grow exponentially using immersive assembly also grow linearly using this technology, and similarly, the roughness is also much lower ( $\sim 2$ - to 10-fold) for LbL films assembled in this way (49).

Spin assembly typically produces significantly more organized films and multilayers than immersive assembly, which has made it a useful tool in preparing optical coatings with 
controllable and homogenous color (14) and for preparing transparent films (44). Similarly, spin assembly is useful for preparing LEDs with higher luminance than immersive assembly (41). A primary limitation for spin assembly in terms of application is that it is limited to coating small planar substrates, as increasing the substrate size requires higher spin speeds. Furthermore, spin coating non-planar surfaces is complicated.

In summary, spin assembly uses rotating substrates to deposit layers and remove excess coating material. Spin assembly typically produces thinner and more organized/stratified multilayers, and the process can be significantly quicker, than immersive assembly. The spin coater needed for assembly is commonly accessible in many research environments, and even some industrial settings, such as with the robotic wafer processing common in the semiconductor industry, which could facilitate translation from the laboratory to real-world applications. Furthermore, depositing multilayer films on non-flat surfaces, or even flat but rough surfaces, can be challenging due to the shear forces involved with film assembly. Nevertheless, the film and process properties arising from spin assembly, including smooth films assembled in a relatively short time, continue to make this method an attractive choice.

\section{Spray Assembly}

Spray LbL assembly is another assembly category, where films are assembled by aerosolizing polymer solutions and sequentially spraying them onto substrates (16). Although spraying air has been used to dry films during LbL assembly to reduce contamination (and align carbon nanotubes) (50), here we discuss spray assembly solely in the context of layer deposition. Standard spray assembly is much faster (as quick as $\sim 6$ seconds per layer) than immersive assembly (51), and approaches an industrial level far surpassing spin assembly (52, 53). Vacuum can also be used to further speed up the process by minimizing the lag time between spraying and washing, and vice versa, and to facilitate the spray coating of three-dimensional objects like membranes (54).

In spray assembly, the film properties, such as the morphology, uniformity, chemical composition, and selective membrane properties can be tailored to be similar to those prepared by immersive assembly, with the film thickness influenced by suspension concentration, spray flow rate, spray duration, resting duration, whether or not the substrate is washed and for how 
long, and whether the solution is sprayed vertically or horizontally $(16,51,55-57)$. This control arises from the two main forces governing the spray assembly process, namely bulk movement in the actual spray, and random movement in the liquid film (56). The random movement in the liquid film allows for polymer rearrangement and generates much higher convection close to the substrate, allowing for improved deposition. This is because of the submicron thickness of the liquid film at the substrate interface and because of the speed at which the spray contacts the substrate $(16,56)$. Interestingly, washing the substrate generally produces thicker films than leaving the substrate unwashed, due to polymer rearrangements during washing (51). Like spin assembly, the films resulting from spray assembly have more distinct layers in comparison to immersive assembly $(16,53)$.

Spray assembly has also been combined with other technologies to leverage technologyspecific advantages and automate the assembly process. For example, a disadvantage of spray LbL assembly is that the obtained films may not be homogeneous due to the effects of gravity draining, causing increased deposition in the vicinity of the solution drips, and because of irregular patterns caused by the spray nozzles at certain distances $(51,57)$. To address this problem, rotating the substrate during spray assembly allows for the preparation of more homogeneous films and sub-second spray times for each layer $(56,58,59)$. By spraying rotating substrates, a majority of the polymer added to the substrate is adsorbed. In comparison, the vast majority of polymer remains in the coating solutions after immersive assembly. Therefore, applicable concentrations roughly 10-50 times less than those required for immersive assembly can be used for spray assembly on rotating substrates $(55,59)$. Larger three-dimensional substrates, such as tubular membranes, can also be coated by rotating the substrate during spraying (60). A further improvement has been the computer-aided automation of spray assembly to reduce manual processing $(58,60)$. Similar to the use of automated immersive assembly on QCM chips, the use of QCM chips for automated spray assembly enables feedback loop control and tracking of real-time film growth (61). Automated spray assembly has also been combined with roll-to-roll processing for coating industrially relevant sized substrates tens of meters long (62). Roll-to-roll spray assembly can also be used to coat particulate substrates with multilayer films by performing spray assembly on particulate substrates immobilized on top of a dissolvable surface (63). 
A stand-alone spray assembly technology for coating particulate substrates utilizes surface acoustic waves of 1-10 nm in amplitude to atomize polymers and cargo (17). As the atomized droplets move through the air, the solvent evaporates and the polymer condenses into particle form, resulting in the first atomized solution becoming the template for subsequent coatings, with $\sim 1000$ carriers produced from each microliter of solution. The particles are dialyzed to remove excess polymer and then added to a solution of oppositely charged polymer, and re-atomized to coat the particles. This process can be repeated for multilayer assembly, however the dialysis process increases the processing time of this technology to roughly 24 hours for each layer.

Spray assembly has found use for a wide variety of applications because it can be used to coat industrially relevant substrate sizes with relative ease (62), and is not limited to planar substrates $(54,60,63)$. It has been used to prepare flame retardant films over cotton cloth, where it was shown that spraying on vertically oriented substrates produced superior flame retardant films compared with both spraying on horizontally oriented substrates or dipping (57). Clothing material was also coated with spray assembly to control air flux and provide chemical protection potentially for use with military uniforms (54). Like other assembly technologies, spray assembly has been used to prepare antireflective coatings (61), and similarly car tinting with structured coloring to reduce heating from infrared light (62). Membrane tubes could also be coated to improve the separation of organic dyes from water (60). Because the structure of the films can be controlled at the nanometer-level by the spray time, spray assembly can be used to control conductance in thin films in ways which are not available to other assembly technologies (59). Spray assembly on particles has been used to monitor cellular uptake of different coatings and aspect ratios of particles (63), and has been used for gene delivery in vitro (17). The primary use of spray assembly for applications and industry relate directly to the rapid assembly times, and because this technology is amenable to both automation and scale up.

In summary, spray assembly produces multilayer films by aerosolizing coating solutions and spraying them onto the substrate. The resulting films are typically well organized with distinct layers. Spray assembly is a quick and easy method to coat large or non-planar substrates, although immersive assembly remains the method of choice for coating complex 3D substrates. Spray assembly is one of the most highly relevant technologies for industrial applications, as it is already widely used industrially. 


\section{Electromagnetic Assembly}

Electromagnetic assembly is based on the use of an applied electric or magnetic field to effect layering, such as by coating electrodes in polymer solutions or by moving magnetic particulate substrates in and out of coating solutions $(18,19)$. The former, commonly referred to as electrodeposition, is a well-established technology for coating materials using an applied voltage in electrolytic cells. In the standard electrodeposition setup, two electrodes are immersed in polymer solution followed by applying an electric current. The electrodes are then washed and placed into solution of an oppositely charged polymer; the polarities of the electrodes are reversed and the process is repeated (64). Electrodeposition can be used to rapidly assemble ions, polymers and colloids in significantly less time than immersive assembly (18). For example, bimetallic mesoporous LbL films can be prepared by electrodeposition, with the electrodeposition time determining the layer thickness at $1.5 \mathrm{~nm}$ per second (65). In another setup the substrate can be placed between the two electrodes, allowing for planar substrates to be coated (66), or even immobilized particles (13). This technology results in films roughly twice the thickness of centrifugation-based assembly for immobilized particulate substrates (13), or immersive assembly for planar substrates (66). Electrodeposition can also use higher voltages, upwards of $30 \mathrm{~V}(13,66)$; however, the assembly process for immobilized particles can take as long as 15 min per layer (13).

The thickness of the electrodeposited films are directly related to the voltage used during assembly, with the optimum voltage for achieving the thickest films dependent on the $\mathrm{pH}$ of the polymer solution (67). Higher voltages can cause desorption of the film as the electrode/substrate begins to repel the previously deposited layer. Generally, $\mathrm{pH}$ values lower than the $\mathrm{p} K_{\mathrm{a}}$ of the polymers need lower voltages to reach peak thickness, and that peak thickness is also larger than the peak thickness at higher $\mathrm{pH}$, closer to the $\mathrm{p} K_{\mathrm{a}}$ or above the $\mathrm{p} K_{\mathrm{a}}$ of the polymers. However, if the voltage is raised high enough, a secondary peak thickness can be reached, allowing for the assembly of films at $\mathrm{pH}$ values otherwise difficult to grow using other technologies (67). The reason for this valley in thickness is that at high voltages, the electrolysis of water at the electrode plays a bigger role in hindering polymer adsorption; however, at even higher voltages $(>3 \mathrm{~V})$ the electrostatic interaction between the polymer and electrode exceeds any hindrance 
due to electrolysis (67). For example, polymer/enzyme films were roughly twice as thick, when assembled at an optimal voltage of $1.2 \mathrm{~V}$ compared with using lower or higher voltages below 3 $\mathrm{V}$ (68). These studies show that electrodeposition is similar to other LbL assembly technologies in the sense that the $\mathrm{pH}$ of the polymer solution, and therefore the configuration of the polymer itself, is crucial in controlling film thickness.

At higher voltages, electromagnetically assembled films are more interpenetrated than immersive-assembled films, which is in contrast to the highly stratified films prepared at lower voltages (66). When forming polymer/polymer films the refractive index does not change significantly during film growth in a flow cell under an electric current, suggesting a more homogenous deposition than immersive assembly (69). Similarly, polymer/colloid films show high organization as the refractive index decreases and transmittance increases when assembling films under higher voltages (70). Correspondingly, electrodeposited enzyme/polymer films are more uniform than immersive-assembled films, with 90\% coverage of the substrate versus $50 \%$ coverage (68). Because of this stratification and high surface coverage, electrodeposition allows for control over the spacing between layers (68).

Electrodeposition can also be achieved by using local effects at the electrodes, such as inducing redox reactions or changes in $\mathrm{pH}$. The $\mathrm{pH}$ of the solution near the anode and cathode changes significantly from bulk solution to lower and higher $\mathrm{pH}$ values, respectively (71). The low $\mathrm{pH}$ near the anode can induce polymer deposition, although this $\mathrm{pH}$-induced electrodeposition is fairly limited, as only a few bilayers can be deposited (using materials like alginate and chitosan) because the layers become too thick (tens of micrometers) for the electric current to penetrate, resulting in no $\mathrm{pH}$ change and therefore no deposition. Using a similar principle, covalently stabilized films can be prepared by generating copper(I) from copper(II) in situ at the electrode/substrate for crosslinking azide- and alkyne-containing polymers with copper-catalyzed “click” reactions (20). Polymers containing electrically sensitive click reactions can also be electrocoupled, allowing for $500 \mathrm{~nm}$ transparent, and therefore stratified and homogenous films to be prepared in $30 \mathrm{~min}$ (72). One-pot synthesis can be performed using the same basic principles by switching between oxidative and reductive reactions by alternating the voltage, allowing for wash-free assembly using electropolymerization (73). 
Magnets, rather than electric currents, can be used to assemble LbL films on sensitive particulate templates, such as emulsions (74), or small templates difficult to pellet through centrifugation, such as sub-10 nm iron oxide nanoparticles (19). Template particles containing magnetic nanoparticles can be separated from polymer solution using a magnet, which, similar to the filtration method, allows for nearly $100 \%$ of the particles to be recovered in a centrifugationfree LbL assembly process (75). The use of magnets or an external field can also be used to orient layered magnetic nanoparticles on a planar substrate so that a subsequent layer of nanoparticles can deposit more rapidly and in an oriented fashion (76). This technology uses standard immersive assembly for the deposition of positively and negatively charged magnetic nanoparticles with application of a magnetic field between deposition steps. Therefore, the thickness does not increase in relation to standard immersive assembly; however, the absorbance of the film increases with application of the magnet, suggesting increased packing (76).

Electromagnetic assembly has found use in several different applications, as it can be used to form LbL films with unique compositions that are not readily assembled using other technologies. Bimetallic films of Pt and Pd layers have Brunauer-Emmett-Teller surface areas of $\sim 40 \mathrm{~m}^{2} \mathrm{~g}^{-1}$, and therefore exhibit enhanced electrochemical activity in the methanol oxidation reaction compared with single-layer films (65). Antireflective coatings can be prepared by adjusting the refractive index of the films by assembling the films at different voltages (70). Biological applications have also been explored, as biocompatible coatings can be formed using electromagnetic assembly, with in vitro cytotoxicity tests confirming negligible cytotoxicity (71). Bienzyme films with bioelectric catalytic properties have higher surface coverage, and therefore activity, when compared with traditionally prepared films (68). The stratification of the assembled films is also conducive toward high-performance photoelectric devices (72), and separation membranes (66). Hollow polymer capsules (from micrometers to sub-100 nm in diameter), can also be prepared using electrodeposition on immobilized particles (13).

In summary, electromagnetic assembly uses electric or magnetic fields, typically in the form of electrodes in polymer solutions or magnetic particulate substrates, to deposit films. Electromagnetic assembly can exploit current-induced changes in $\mathrm{pH}$ or redox-reactions to effect film assembly, thus using a driving force significantly different from that of the other main assembly categories. Generally, electromagnetic-assembled films are thicker/more densely packed than films prepared using other LbL assembly methods $(13,68)$. Electromagnetic 
assembly is still not as common as some of the other technologies, and even though it requires special equipment and expertise, it does offer a different approach to multilayer film assembly (e.g., through magnetic handling of substrates and materials or through electrically induced assembly), thereby providing unique opportunities for assembling films.

\section{Fluidic Assembly}

Fluidic assembly can be used to deposit multilayers using fluidic channels, both by coating the channel walls, and by coating a substrate placed or immobilized in a fluidic channel (77). The general method involves using pressure or vacuum to sequentially move polymer and washing solutions through the channels, which can be fluidic components such as tubing or capillaries, or designed microfluidic networks $(78,79)$. Flow-chamber based QCM is a common fluidic assembly technology used for investigating thin film properties and multilayer growth by providing crucial real-time information (22). Higher concentrations of polymer solution typically yield thicker films (79), with the contact time, rather than the flow rate, being the crucial factor determining the amount of adsorbed polymer under flow (80).

Fluidic assembly is typically implemented using a pump, capillary forces, or spinning to transport the liquid through the channels, although pipetting and static incubation can also be used. However, fluidic assembly strongly resembles immersive assembly when polymer solutions are allowed to remain in static contact with the substrate for greater than 10 min (81, 82). Polymer and washing solutions loaded into channels with a pump or vacuum can deposit 1.5 nm thick layers in 5-10 min (83). Capillary forces can also be used to pull polymer solutions through microfluidic channels by placing droplets of solution at fluidic inlets followed by spinning the substrate to remove the solution, allowing for $\sim 1.2 \mathrm{~nm}$ thick layers to be deposited in less than 2 min (84). Fluidic layering based on capillary forces is easy to implement, as capillary action does not require external active components, but it is not suitable for larger volumes or when dynamic control over the flow rate is needed.

Fluidic devices and perfusion chambers can also be used to achieve region-specific fluidic assembly or to perform fluidic assembly on more complicated 3D structures. For example, complex automated microfluidic devices can be used to assemble hundreds of layers in parallel using capillary flow and vacuum to fill and empty multiple channels (85). This enables 
the high-throughput screening of film libraries using small quantities of materials, as only a droplet is needed to fill a single microchannel. Region-specific films can be coated on substrates by affixing a geometric chamber over the substrate and then flowing the solution through the chamber and over the substrate (80). Perfusion chambers can be used for fluidic layering on complex 3D substrates such as sensitive biological substrates, like arteries, which must remain constantly hydrated during layering (86). Similarly, perfusion chambers can be used to hold agarose that contains immobilized particles for fluidic assembly (87). This technology not only allows for the deposition of polymers but also for the deposition of larger cargo, such as gold nanoparticles or liposomes, and produces films with nearly identical thickness to those prepared by standard centrifugation-based assembly (87).

Vacuum is typically used with other assembly technologies, such as spray assembly, or to remove the solution from channels in fluidic assembly, but it can also be used to form multilayers in a macrofluidic-type assembly, especially on unique substrates like aerogels. Aerogels can be functionalized using vacuum assembly by pouring solutions of conducting polymers, biomolecules or carbon nanotubes from the top and applying vacuum to pull these solutions down through the aerogel (88). Vacuum assembly can also be used to deposit materials that would otherwise be challenging to create uniform multilayers with, such as reducedgraphene oxide (89). For particulate substrates, vacuum assembly can be performed using separating filters, down to $200 \mathrm{~nm}$ in pore size, for centrifugation-free layering $(21,90)$. Vacuum is not applicable for all sensitive templates; however, for template particles including emulsions (91), cell islets (92), or calcium carbonate nanowires (93), a slight vacuum of 100 mbar can facilitate the layering process (21). Less than $3 \%$ of the particles are lost during assembly using an optimized procedure, which is far less than the calculated saturation-based method where $50 \%$ can be lost, or the centrifugation-based method where over $80 \%$ can be lost at high layer numbers (21). This combined filter and vacuum assembly technology yields a layer thickness of $\sim 1.3 \mathrm{~nm}$ and a surface roughness of $\sim 5-10 \mathrm{~nm}$, which are both similar to those prepared via centrifugation-based assembly (94). A filtration setup has also been automated for coating cell islets, using a feedback loop for evacuating the fluid from the reaction chamber, thereby reducing the manual handling time by 60\% (95).

Like vacuum assembly, fluidic assembly is not restricted to planar substrates and is a viable alternative for centrifugation-free assembly on particulate substrates (96-98). Many 
fluidic assembly approaches coat emulsions or liquid crystals, as these are materials well studied in the fluidics field. Generally, the coating and washing solutions are deflected past the flow of particles by using physical gaps smaller than the particles so that the flow can enter perpendicular (96) or parallel to the particle flow stream (97). For fluidic assembly in parallel flow systems, larger template particles ( $50 \mu \mathrm{m}$ in diameter) are necessary, as they can be deflected in a zig-zag pattern using solid pillars at a $\sim 45^{\circ}$ angle to three parallel laminar flow streams: solution A, washing, solution B. This gives a layer thickness of 2-3 nm (97). A similar technology can be used to coat $15 \mu \mathrm{m}$ beads with avidin and biotin, where higher deflection angles have a high correlation to failure rates, with angles of $1^{\circ}$ optimal for a failure rate of virtually zero (99). Instead of pillars, specific geometries can be used to catch emulsions for the fluidic assembly of lipid layers (98). For coating lipid particles with polymers, tangential flow filtration can be used as a type of expedited dialysis for removing excess polymer solution (100). To coat sufficiently large/dense particles, a setup based on fluidized beds can be used. This allows for $\sim 8$ times faster assembly when compared with centrifugation-based assembly and produces films twice as thick (23). In this instance, the force of the washing or polymer solution lifting the particles is balanced against the force of gravity sedimenting the particles, resulting in a fluidized bed where washing and polymer solutions can be pushed past the particles. A similar setup can be used to coat larger $(>100 \mu \mathrm{m})$ particles in packed columns, although these beds do not need to be fluidized due to the large particle size, and gravity rather than a vacuum or pumpdriven fluidics can be used to pull the coating and washing solutions through the column (1).

Numerous applications have been introduced during the process of developing novel technologies for fluidic assembly. Many applications are realized inside capillaries such as improved electrophoresis capillaries (81). Fluidic assembly can also be used to engineer complex flow patterns, such as having flow in opposite directions in the same capillary, simply by changing the outer coating of the capillary walls and generating flow with an electric current (82). Fluidic assembly is not limited to planar substrates, for example multilayer coatings can be prepared on aerogels resulting in improved compressive strength, wet state super elasticity, fluorescence and mechano-responsive resistance, while also creating high charge-storage capacity (88). Damaged aortic porcine arteries can be repaired ex vivo with fluidic assembly, to protect the artery against unwanted blood coagulation, as well as to facilitate healing (86). Similarly, catheter tubing can be coated with anti-fungal multilayers to reduce fouling (78). 
Chromatography beads coated with multilayers of particles increase the surface area of the beads, thereby improving chromatography (1). Although fluidic assembly is typically performed on larger particles (tens or hundreds of micrometers in diameter), smaller particles (below $~ 5$ $\mu \mathrm{m}$ in diameter) can be coated and loaded with functional cargo for potential drug delivery applications by combining microfluidics with immobilization (87). Fragile particulate substrates like emulsions can also be coated with lipids using fluidic assembly for the generation of “synthetic cells” (98). Neuronal cells can be patterned with fluidic assembly (83), and cell islets can be coated to improve robustness, allowing for in vivo transplantation $(92,95)$. Fluidic assembly functions as a valuable tool for coating sensitive particulate substrates, like mammalian cells, that may be damaged using other technologies, such as during handling in centrifugationbased assembly.

In summary, fluidic assembly provides the means to assemble multilayers on surfaces not easily accessible to other methods (e.g., inside capillaries), provides new ways for regionspecific patterning (e.g., by masking a surface with a fluidic channel), and increases the industrial capacity of multilayer assemblies (e.g., through parallelization of film assembly and decrease of reagent consumption). Although the specialized equipment and expertise required to set up (micro)fluidic systems can complicate the use of fluidic assembly, these unique advantages make it attractive for many applications.

\section{Challenges Both Big and Small}

Over the last two decades LbL assembly has undergone an explosive growth in usable materials and substrates, and when taken together with all of the different assembly technologies available, it becomes obvious why LbL assembly is prevalent across a broad spectrum of disciplines. Despite this extensive toolbox, relatively few multilayer films have had widespread impact outside of research environments. One focus for industrial applications is likely the identification of reliable, scalable and resource-effective assembly processes, although this may require different approaches for macroscopic substrates and for microscopic particulate substrates.

For macroscopic substrates, improved high-throughput assembly methods for conformal coatings will play a key role. Immersive and/or spray roll-to-roll assembly is industrially relevant but only readily applicable to flexible planar substrates, therefore innovation in systems that can 
be easily scaled for coating large or numerous 3D macroscopic substrates is needed. Similarly, reducing material waste during the coating process remains important, especially for valuable coating materials like biomolecules and custom polymers. Another challenge for films intended for in vivo biomedical application, such as drug delivery and tissue engineering, is ensuring sterility of the product. This is typically achieved through sterilization (heat, ultraviolet light, chemical treatment, etc.) just prior to use, which can affect film properties and performance. Finally, increasing the reliability and reproducibility of the films, for example by increasing automation and reducing manual intervention, is crucial for extending knowledge about film properties and assembly technologies, and also for applying the multilayer films in real-life applications.

Similar challenges exist for particulate substrates. One crucial difference is that several particulate assembly methods depend on centrifugation, which remains difficult to scale or combine with minimal-intervention high-throughput assembly. Furthermore, yield and size ranges need to be specified for the various technologies, as these details are often not determined. Detailed film properties also need to be investigated so that further comparisons between planar and particulate substrates can be drawn, such as layer interpenetration, layer density, film stability or responsiveness, and permeability, which have primarily been studied on planar substrates. Altogether, these challenges are not trivial and require focused efforts to overcome; they are also not unique to the field of LbL assembly. One way to address these challenges is to continue to be open and look for solutions in new and sometimes unexpected areas, both in neighboring and more distant fields, which has underpinned much of the technological innovation in LbL assembly.

\section{Opportunities: Thinking Outside the Box}

LbL assembly is a firmly established technology and shows great promise in multiple, diverse fields. Much of the development up until now has been on utilizing new molecular driving forces for film assembly, thus enabling the use of a suite of substrates and layer materials. However, this enormous potential still remains largely limited to small-scale research settings and requires technological and methodological innovation. Despite a surge of new technologies, many being recent developments, unmet challenges still remain; both for 
harnessing the specific strengths of different technologies for specific applications, and also for developing new and improved technologies.

Although significant work has been undertaken on establishing new assembly technologies, there are only a few studies that have chosen a specific assembly technology for the material properties generated (e.g., stratification, density, roughness), rather than the processing properties utilized (e.g., ease-of-use, material and time savings, lowered involvement, larger batches). For example, for applications where electrical conductivity is important (such as fuel cells and batteries), the conductivity of an immersive-assembled film can be superior to that of spray-assembled films, which can be explained by differences in the interlayer organization of the constituent conductive layer materials (interpenetration versus stratification) (59). Conversely, for applications where optical clarity and/or wetting behavior is important, spinassembly can allow for an optically transparent film with well-controlled water contact-angles to be assembled due to the smooth, stratified layers formed, while an immersive-assembled film can be translucent and with a contact angle that drastically changes depending on the layer deposited due to the rough, interpenetrated layers formed (Fig. 3) (44). However, layer structure is only one of the critical film properties to be taken into account when designing films for specific applications. For example, the higher surface coverage and layer density associated with electromagnetic assembly can allow for electrodeposited enzyme films to have higher enzymatic activity than comparable immersive-assembled films (68). Of course, the layer structure and density are not relevant if the desired film components cannot be layered, which can be an issue, for example, when using materials with low charge density (e.g., reduced-graphene oxide) or with a low surface area of contact (e.g., branched nanowires). In such cases, technologies such as dewetting and vacuum assembly enable film formation using constituents that cannot easily be layered using other technologies $(8,89)$. These examples demonstrate how judicious choice of assembly technology can enable the assembly of new and improved thin films. As our understanding of the different technologies and how they compare to each other increases, so does the opportunity to let this insight help guide the development of the next generation of LbL assembled thin films.

It is noteworthy that the assembly technologies discussed herein were not originally developed for LbL assembly, and cross-over technologies from other fields will continue to play an important part for new, and perhaps even revolutionary, developments. One interesting 
example involving industrial scale layering was performed using a modified car wash for spray assembly on a full-sized car (101). Technologies long used in the pharmaceutical industry, such as methods used to treat, purify and concentrate pharmaceuticals, may prove transformative for biomedical applications. Similarly, using everyday objects like spray-paint cans could revolutionize assembly methods by essentially combining dewetting and spray assembly, for rapid region-specific assembly with little to no material waste and no washing steps. Other combinations between existing assembly technologies should also help expedite and automate the assembly process. Along these lines, technologies for assembly on particulate substrates are expected to continue to integrate immobilization methods, as they allow collections of particles to be treated like planar substrates, making accessible many of the planar assembly technologies discussed herein. Another promising approach for particulate substrates could be to use a type of "sponge" to adsorb excess polymer from solution, thus removing the need to pellet the particles. In terms of future developments for applications, it will be important to understand the interaction between multilayer films and complex and natural environments, such as those found in the human body (40), outdoors, or in seawater. An important aspect of this could be the use of functional substrates capable of compounding the benefits of different multilayers in a synergistic fashion. Overall, the future of LbL assembly is bright, and as the black box of assembly technologies is slowly illuminated, great potential for innovation and application will be found. 


\section{References and Notes:}

1. J. J. Kirkland, Porous thin-layer modified glass bead supports for gas liquid chromatography. Anal. Chem. 37, 1458-1461 (1965).

2. R. Iler, Multilayers of colloidal particles. J. Colloid Interf. Sci. 21, 569-594 (1966).

3. G. Decher, Fuzzy nanoassemblies: Toward layered polymeric multicomposites. Science 277, 1232-1237 (1997).

4. P. Ott, K. Trenkenschuh, J. Gensel, A. Fery, A. Laschewsky, Free-standing membranes via covalent cross-linking of polyelectrolyte multilayers with complementary reactivity. Langmuir 26, 18182-18188 (2010).

5. F. Caruso, R. A. Caruso, H. Möhwald, Nanoengineering of inorganic and hybrid hollow spheres by colloidal templating. Science 282, 1111-1114 (1998).

6. E. Donath, G. B. Sukhorukov, F. Caruso, S. A. Davis, H. Möhwald, Novel hollow polymer shells by colloid-templated assembly of polyelectrolytes. Angew. Chem. Int. Ed. 37, 2201-2205 (1998).

7. X. Zhang, H. Chen, H. Zhang, Layer-by-layer assembly: From conventional to unconventional methods. Chem. Commun. 14, 1395-1405 (2007).

8. B. S. Shim et al., Nanostructured thin films made by dewetting method of layer-by-layer assembly. Nano Lett. 7, 3266-3273 (2007).

9. K. Fujimoto, S. Fujita, B. Ding, S. Shiratori, Fabrication of layer-by-layer self-assembly films using roll-to-roll process. Jpn. J. Appl. Phys. 44, L126 (2005).

10. E. Donath et al., Nonlinear hairy layer theory of electrophoretic fingerprinting applied to consecutive layer by layer polyelectrolyte adsorption onto charged polystyrene latex particles. Langmuir 13, 5294-5305 (1997).

11. D. Grigoriev, T. Bukreeva, H. Möhwald, D. Shchukin, New method for fabrication of loaded micro-and nanocontainers: Emulsion encapsulation by polyelectrolyte layer-bylayer deposition on the liquid core. Langmuir 24, 999-1004 (2008).

12. N. G. Hoogeveen, M. A. Cohen Stuart, G. J. Fleer, M. R. Böhmer, Formation and stability of multilayers of polyelectrolytes. Langmuir 12, 3675-3681 (1996).

13. J. J. Richardson et al., Preparation of nano- and microcapsules by electrophoretic polymer assembly. Angew. Chem. Int. Ed. 52, 6455-6458 (2013).

14. I. M. Thomas, Single-layer $\mathrm{TiO}_{2}$ and multilayer $\mathrm{TiO}_{2}-\mathrm{SiO}_{2}$ optical coatings prepared from colloidal suspensions. Appl. Optics 26, 4688-4691 (1987).

15. L. Ma et al., Layer-by-layer self-assembly under high gravity field. Langmuir 28, 98499856 (2012).

16. J. B. Schlenoff, S. T. Dubas, T. Farhat, Sprayed polyelectrolyte multilayers. Langmuir 16, 9968-9969 (2000).

17. A. Qi et al., Template-free synthesis and encapsulation technology for Layer-by-Layer polymer nanocarrier fabrication. ACS Nano 5, 9583-9591 (2011).

18. J. Sun, M. Gao, J. Feldmann, Electric field directed layer-by-layer assembly of highly fluorescent CdTe nanoparticles. J. Nanosci. Nanotechnol. 1, 133-136 (2001).

19. X. Hong et al., Fabrication of magnetic luminescent nanocomposites by a layer-by-layer self-assembly approach. Chem. Mater. 16, 4022-4027 (2004).

20. G. Rydzek et al., Polymer multilayer films obtained by electrochemically catalyzed click chemistry. Langmuir 26, 2816-2824 (2010). 
21. A. Voigt et al., Membrane filtration for microencapsulation and microcapsules fabrication by layer-by-layer polyelectrolyte adsorption. Ind. Eng. Chem. Res. 38, 40374043 (1999).

22. C. Picart et al., Buildup mechanism for poly (L-lysine)/hyaluronic acid films onto a solid surface. Langmuir 17, 7414-7424 (2001).

23. J. J. Richardson et al., Fluidized bed layer-by-layer microcapsule formation. Langmuir 30, 10028-10034 (2014).

24. D. Lee, M. F. Rubner, R. E. Cohen, All-nanoparticle thin-film coatings. Nano Lett. 6, 2305-2312 (2006).

25. G. Decher, J. D. Hong, J. Schmitt, Buildup of ultrathin multilayer films by a selfassembly process: III. Consecutively alternating adsorption of anionic and cationic polyelectrolytes on charged surfaces. Thin Solid Films 210/211, 831-835 (1992).

26. S. T. Dubas, J. B. Schlenoff, Factors controlling the growth of polyelectrolyte multilayers. Macromolecules 32, 8153-8160 (1999).

27. D. Peiffre, T. Corley, G. Halpern, B. Brinker, Utilization of polymeric materials in laser fusion target fabrication. Polymer 22, 450-460 (1981).

28. G. L. Gaines Jr, Deposition of colloidal particles in monolayers and multilayers. Thin Solid Films 99, 243-248 (1983).

29. A. Fou, O. Onitsuka, M. Ferreira, M. Rubner, B. Hsieh, Fabrication and properties of light-emitting diodes based on self-assembled multilayers of poly (phenylene vinylene). J. Appl. Phys. 79, 7501-7509 (1996).

30. S. S. Shiratori, M. Yamada, Nano-scale control of composite polymer films by masscontrolled layer-by-layer sequential adsorption of polyelectrolytes. Polym. Adv. Technol. 11, 810-814 (2000).

31. C. G. Gölander, H. Arwin, J. C. Eriksson, I. Lundstrom, R. Larsson, Heparin surface film formation through adsorption of colloidal particles studied by ellipsometry and scanning electron microscopy. Colloids Surf. 5, 1-16 (1982).

32. Y. Fu et al., Facile and efficient approach to speed up layer-by-layer assembly: Dipping in agitated solutions. Langmuir 27, 672-677 (2011).

33. J. J. Richardson et al., Immersive polymer assembly on immobilized particles for automated capsule preparation. Adv. Mater. 25, 6874-6878 (2013).

34. A. J. Mateos, A. A. Cain, J. C. Grunlan, Large-scale continuous immersion system for layer-by-layer deposition of flame retardant and conductive nanocoatings on fabric. Ind. Eng. Chem. Res. 53, 6409-6416 (2014).

35. J. Li, H. D. H. Stöver, Pickering emulsion templated layer-by-layer assembly for making microcapsules. Langmuir 26, 15554-15560 (2010).

36. H. Liu, X. Gu, M. Hu, Y. Hu, C. Wang, Facile fabrication of nanocomposite microcapsules by combining layer-by-layer self-assembly and Pickering emulsion templating. RSC Adv. 4, 16751-16758 (2014).

37. G. B. Sukhorukov et al., Layer-by-layer self assembly of polyelectrolytes on colloidal particles, Colloids Surf., A, 137, 1998, $253-266$ (1998).

38. K. Szczepanowicz et al., Formation of biocompatible nanocapsules with emulsion core and pegylated shell by polyelectrolyte multilayer adsorption. Langmuir 26, 12592-12597 (2010). 
39. T. G. Shutava, P. P. Pattekari, K. A. Arapov, V. P. Torchilin, Y. M. Lvov, Architectural layer-by-layer assembly of drug nanocapsules with PEGylated polyelectrolytes. Soft Matter 8, 9418-9427 (2012).

40. Y. Yan, M. Björnmalm, F. Caruso, Assembly of layer-by-layer particles and their interactions with biological systems. Chem. Mater. 26, 452-460 (2014).

41. H. Hong, R. Steitz, S. Kirstein, D. Davidov, Superlattice structures in poly (phenylenevinylene)-based self-assembled films. Adv. Mater. 10, 1104-1108 (1998).

42. P. A. Chiarelli et al., Controlled fabrication of polyelectrolyte multilayer thin films using spin-assembly. Adv. Mater. 13, 1167-1171 (2001).

43. J. Cho, K. Char, J. D. Hong, K. B. Lee, Fabrication of highly ordered multilayer films using a spin self-assembly method. Adv. Mater. 13, 1076-1078 (2001).

44. J. Seo, J. L. Lutkenhaus, J. Kim, P. T. Hammond, K. Char, Effect of the layer-by-layer (LbL) deposition method on the surface morphology and wetting behavior of hydrophobically modified PEO and PAA LbL films. Langmuir 24, 7995-8000 (2008).

45. S. Vozar et al., Automated spin-assisted layer-by-layer assembly of nanocomposites. Rev. Sci. Instrum. 80, 023903 (2009).

46. P. A. Patel, A. V. Dobrynin, P. T. Mather, Combined effect of spin speed and ionic strength on polyelectrolyte spin assembly. Langmuir 23, 12589-12597 (2007).

47. E. Kharlampieva, V. Kozlovskaya, J. Chan, J. F. Ankner, V. V. Tsukruk, Spin-assisted layer-by-layer assembly: Variation of stratification as studied with neutron reflectivity. Langmuir 25, 14017-14024 (2009).

48. J. Schmitt et al., Metal nanoparticle/polymer superlattice films: Fabrication and control of layer structure. Adv. Mater. 9, 61-65 (1997).

49. C. Jiang et al., Controlled exponential growth in layer-by-layer multilayers using high gravity fields. J.Mater. Chem. A 2, 14048-14053 (2014).

50. B. S. Shim, N. A. Kotov, Single-walled carbon nanotube combing during layer-by-layer assembly: From random adsorption to aligned composites. Langmuir 21, 9381-9385 (2005).

51. A. Izquierdo, S. Ono, J.-C. Voegel, P. Schaaf, G. Decher, Dipping versus spraying: Exploring the deposition conditions for speeding up layer-by-layer assembly. Langmuir 21, 7558-7567 (2005).

52. M. Dierendonck, S. De Koker, R. De Rycke, B. G. De Geest, Just spray it-LbL assembly enters a new age. Soft Matter 10, 804-807 (2014).

53. P. Schaaf, J. C. Voegel, L. Jierry, F. Boulmedais, Spray-assisted polyelectrolyte multilayer buildup: From step-by-step to single-step polyelectrolyte film constructions. Adv. Mater. 24, 1001-1016 (2012).

54. K. C. Krogman, J. L. Lowery, N. S. Zacharia, G. C. Rutledge, P. T. Hammond, Spraying asymmetry into functional membranes layer-by-layer. Nat. Mater. 8, 512-518 (2009).

55. M. Merrill, C. Sun, Fast, simple and efficient assembly of nanolayered materials and devices. Nanotechnology 20, 075606 (2009).

56. W. D. Mulhearn, D. D. Kim, Y. Gu, D. Lee, Facilitated transport enhances spray layerby-layer assembly of oppositely charged nanoparticles. Soft Matter 8, 10419-10427 (2012).

57. J. Alongi, F. Carosio, A. Frache, G. Malucelli, Layer by layer coatings assembled through dipping, vertical or horizontal spray for cotton flame retardancy. Carbohyd. Polym. 92, 114-119 (2013). 
58. K. C. Krogman, N. S. Zacharia, S. Schroeder, P. T. Hammond, Automated process for improved uniformity and versatility of layer-by-layer deposition. Langmuir 23, 31373141 (2007).

59. F. S. Gittleson, D. J. Kohn, X. Li, A. D. Taylor, Improving the assembly speed, quality, and tunability of thin conductive multilayers. ACS Nano 6, 3703-3711 (2012).

60. H. Q. Tang, S. L. Ji, L. L. Gong, H. X. Guo, G. J. Zhang, Tubular ceramic-based multilayer separation membranes using spray layer-by-layer assembly. Polym. Chem. 4, 5621-5628 (2013).

61. N. Fukao, K.-H. Kyung, K. Fujimoto, S. Shiratori, Automatic spray-LbL machine based on in-situ QCM monitoring. Macromolecules 44, 2964-2969 (2011).

62. K. C. Krogman, R. E. Cohen, P. T. Hammond, M. F. Rubner, B. N. Wang, Industrialscale spray layer-by-layer assembly for production of biomimetic photonic systems. Bioinspir. Biomim. 8, 045005 (2013).

63. S. W. Morton et al., Scalable manufacture of built-to-order nanomedicine: Spray-assisted layer-by-layer functionalization of PRINT nanoparticles. Adv. Mater. 25, 4707-4713 (2013).

64. P. R. Van Tassel, Polyelectrolyte adsorption and layer-by-layer assembly: Electrochemical control. Curr. Opin. Colloid In. 17, 106-113 (2012).

65. H. Wang, S. Ishihara, K. Ariga, Y. Yamauchi, All-metal layer-by-layer films: Bimetallic alternate layers with accessible mesopores for enhanced electrocatalysis. J. Am. Chem. Soc. 134, 10819-10821 (2012).

66. G. Zhang, L. Dai, L. Zhang, S. Ji, Effects of external electric field on film growth, morphology, and nanostructure of polyelectrolyte and nanohybrid multilayers onto insulating substrates. Langmuir 27, 2093-2098 (2011).

67. Y. H. Ko et al., Electric-field-assisted layer-by-layer assembly of weakly charged polyelectrolyte multilayers. Macromolecules 44, 2866-2872 (2011).

68. L. Shi et al., Site-selective lateral multilayer assembly of bienzyme with polyelectrolyte on ITO electrode based on electric field-induced directly layer-by-layer deposition. Biomacromolecules 4, 1161-1167 (2003).

69. A. P. Ngankam, P. R. Van Tassel, In situ layer-by-layer film formation kinetics under an applied voltage measured by optical waveguide lightmode spectroscopy. Langmuir 21, 5865-5871 (2005).

70. Y. Omura, K. Kyung, S. Shiratori, S. Kim, Effects of applied voltage and solution $\mathrm{pH}$ in fabricating multilayers of weakly charged polyelectrolytes and nanoparticles. Ind. Eng. Chem. Res. 53, 11727-11733 (2014).

71. Z. Wang et al., Electrodeposition of alginate/chitosan layer-by-layer composite coatings on titanium substrates. Carbohyd. Polym. 103, 38-45 (2014).

72. M. Li et al., Electrochemical-coupling layer-by-layer (ECC-LbL) assembly. J. Am. Chem. Soc. 133, 7348-7351 (2011).

73. M. Li et al., In situ switching layer-by-layer assembly: One-pot rapid layer assembly via alternation of reductive and oxidative electropolymerization. Chem. Commun. 49, 68796881 (2013).

74. B. Mu, P. Liu, P. Du, Y. Dong, C. Lu, Magnetic-targeted pH-responsive drug delivery system via layer-by-layer self-assembly of polyelectrolytes onto drug-containing emulsion droplets and its controlled release. J. Polym. Sci. A Polym. Chem. 49, 19691976 (2011). 
75. R. Wilson, D. G. Spiller, I. A. Prior, R. Bhatt, A. Hutchinson, Magnetic microspheres encoded with photoluminescent quantum dots for multiplexed detection. J. Mater. Chem. 17, 4400-4406 (2007).

76. S. Dey, K. Mohanta, A. J. Pal, Magnetic-field-assisted layer-by-layer electrostatic assembly of ferromagnetic nanoparticles. Langmuir 26, 9627-9631 (2010).

77. Y. Wang et al., Coupling electrodeposition with layer-by-layer assembly to address proteins within microfluidic channels. Adv. Mater. 23, 5817-5821 (2011).

78. N. Raman, M.-R. Lee, S. P. Palecek, D. M. Lynn, Polymer multilayers loaded with antifungal $\beta$-peptides kill planktonic Candida albicans and reduce formation of fungal biofilms on the surfaces of flexible catheter tubes. J. Control. Release 191, 54-62 (2014).

79. N. Madaboosi et al., Microfluidics as a tool to understand the build-up mechanism of exponential-like growing films. Macromol. Rapid Comm. 33, 1775-1779 (2012).

80. H.-J. Kim, K. Lee, S. Kumar, J. Kim, Dynamic sequential layer-by-layer deposition method for fast and region-selective multilayer thin film fabrication. Langmuir 21, 85328538 (2005).

81. H. Katayama, Y. Ishihama, N. Asakawa, Stable cationic capillary coating with successive multiple ionic polymer layers for capillary electrophoresis. Anal. Chem. 70, 5272-5277 (1998).

82. S. L. Barker, D. Ross, M. J. Tarlov, M. Gaitan, L. E. Locascio, Control of flow direction in microfluidic devices with polyelectrolyte multilayers. Anal. Chem. 72, 5925-5929 (2000).

83. D. R. Reyes, E. M. Perruccio, S. P. Becerra, L. E. Locascio, M. Gaitan, Micropatterning neuronal cells on polyelectrolyte multilayers. Langmuir 20, 8805-8811 (2004).

84. H. Jang, S. Kim, K. Char, Multilayer line micropatterning using convective self-assembly in microfluidic channels. Langmuir 19, 3094-3097 (2003).

85. S. A. Castleberry, W. Li, D. Deng, S. Mayner, P. T. Hammond, Capillary flow layer-bylayer: A microfluidic platform for the high throughput assembly and screening of nanolayered film libraries. ACS Nano 8, 6580-6589 (2014).

86. B. Thierry, F. M. Winnik, Y. Merhi, M. Tabrizian, Nanocoatings onto arteries via layerby-layer deposition: Toward the in vivo repair of damaged blood vessels. J. Am. Chem. Soc. 125, 7494-7495 (2003).

87. J. J. Richardson et al., Convective polymer assembly for the deposition of nanostructures and polymer thin films on immobilized particles. Nanoscale 6, 13416-13420 (2014).

88. M. Hamedi et al., Nanocellulose aerogels functionalized by rapid layer-by-layer assembly for high charge storage and beyond. Angew. Chem. Int. Ed. 52, 12038-12042 (2013).

89. B.-S. Kong, J. Geng, H.-T. Jung, Layer-by-layer assembly of graphene and gold nanoparticles by vacuum filtration and spontaneous reduction of gold ions. Chem. Commun. 16, 2174-2176 (2009).

90. S. Hirsjärvi, L. Peltonen, J. Hirvonen, Layer-by-layer polyelectrolyte coating of low molecular weight poly (lactic acid) nanoparticles. Colloids Surf. B 49, 93-99 (2006).

91. A. V. Sadovoy, M. V. Kiryukhin, G. B. Sukhorukov, M. N. Antipina, Kinetic stability of water-dispersed oil droplets encapsulated in a polyelectrolyte multilayer shell. Phys. Chem. Chem. Phys. 13, 4005-4012 (2011).

92. J. T. Wilson et al., Cell surface engineering with polyelectrolyte multilayer thin films. $J$. Am. Chem. Soc. 133, 7054-7064 (2011). 
93. T. Sasaki, M. Shimizu, Y. Wu, K. Sakurai, Chitosan derivatives/calcium carbonate composite capsules prepared by the layer-by-layer deposition method. J. Nanomater. 2008, 1-8 (2008).

94. S. Leporatti et al., Scanning force microscopy investigation of polyelectrolyte nano- and microcapsule wall texture. Langmuir 16, 4059-4063 (2000).

95. J. M. Mets, J. T. Wilson, W. Cui, E. L. Chaikof, An automated process for layer-by-layer assembly of polyelectrolyte multilayer thin films on viable cell aggregates. Adv. Healthc. Mater. 2, 266-270 (2013).

96. C. Priest et al., Microfluidic polymer multilayer adsorption on liquid crystal droplets for microcapsule synthesis. Lab Chip 8, 2182-2187 (2008).

97. C. Kantak, S. Beyer, L. Yobas, T. Bansal, D. Trau, A 'microfluidic pinball' for on-chip generation of layer-by-layer polyelectrolyte microcapsules. Lab Chip 11, 1030-1035 (2011).

98. S. Matosevic, B. M. Paegel, Layer-by-layer cell membrane assembly. Nat. Chem. 5, 958963 (2013).

99. R. D. Sochol et al., in Solid-State Sensors, Actuators and Microsystems Conference (TRANSDUCERS), 2011 16th International. (IEEE, 2011), pp. 1761-1764.

100. S. Hirsjärvi, Y. Qiao, A. Royere, J. Bibette, J.-P. Benoit, Layer-by-layer surface modification of lipid nanocapsules. Eur. J. Pharm. Biopharm. 76, 200-207 (2010).

101. K. Ariga et al., Layer-by-layer nanorchitectectonics: invention, innovation, and evolution. Chem. Lett. 43, 36-68 (2014).

\section{Acknowledgments:}

This work was supported by the Australian Research Council (ARC) under the Australian Laureate Fellowship (F.C., FL120100030) and the Australian Government through an Australian Postgraduate Award (M.B.). This work was also supported by the ARC Centre of Excellence in Convergent Bio-Nano Science and Technology (Project Number CE140100036). 
Image captions (image files supplied separately)

Fig 1. (A) Schematic overview of LbL assembly and (B) an overview showing that the assembly technology influences film and process properties, and application areas.

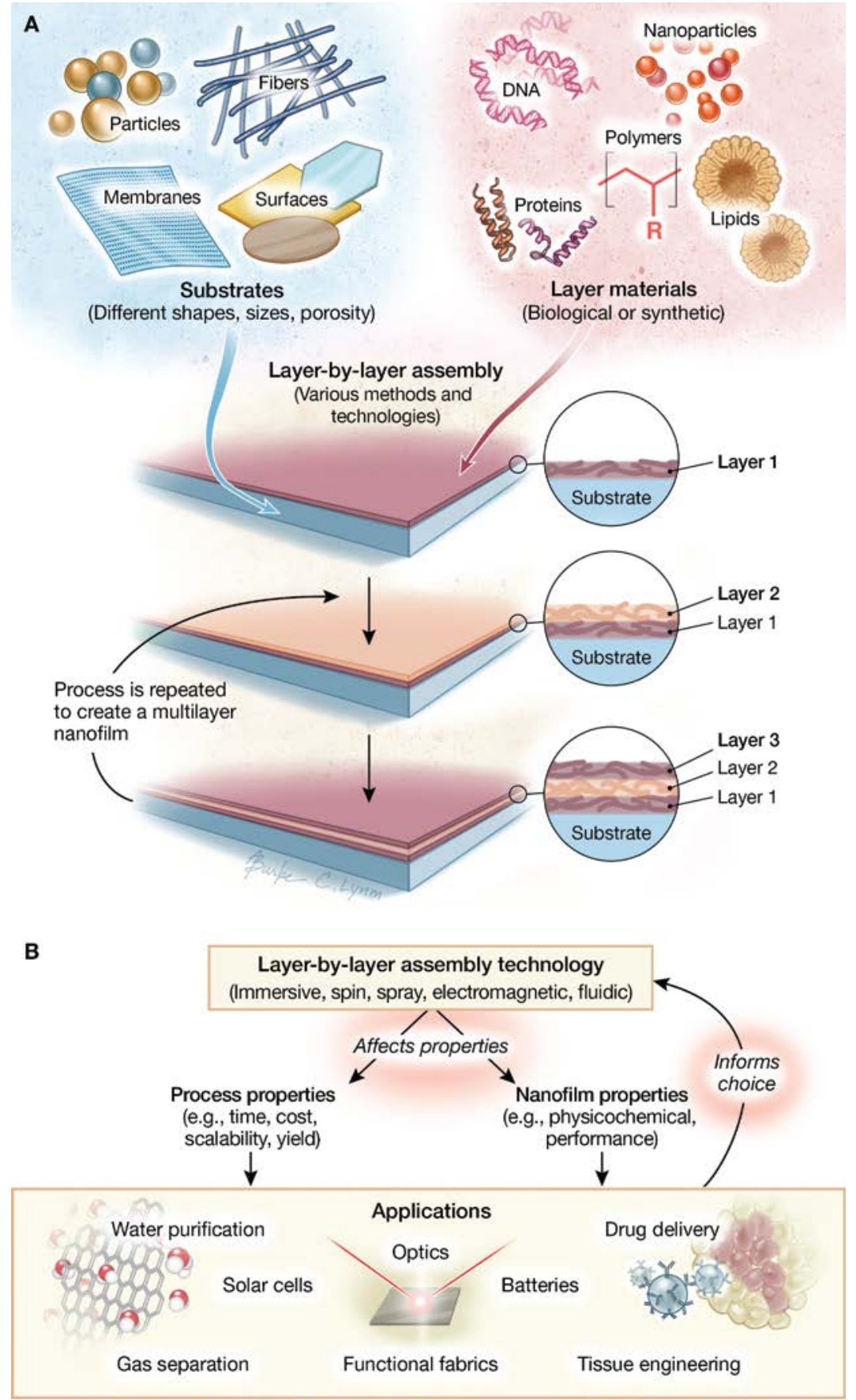


Fig. 2. (A-E) Schematics of the five major technology categories for layer-by-layer assembly.
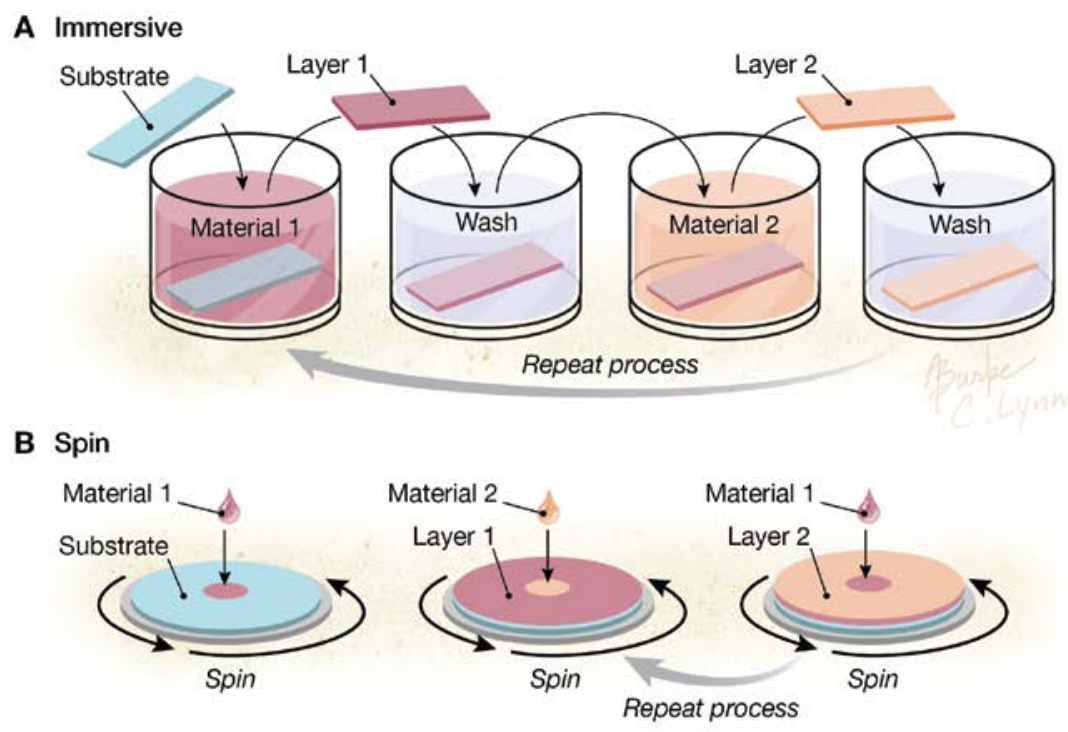

\section{Spray}

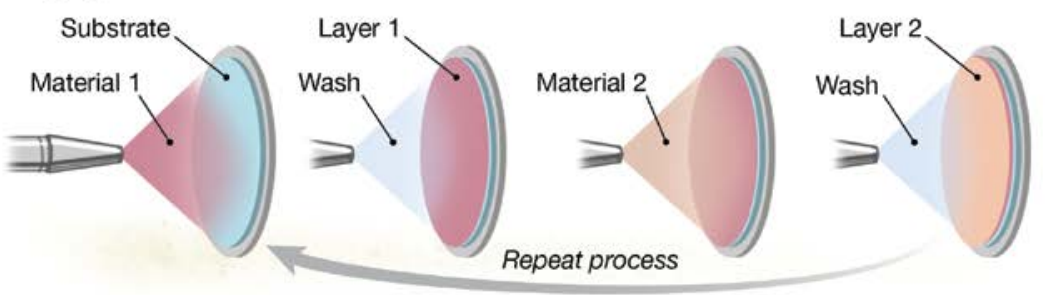

D Electromagnetic

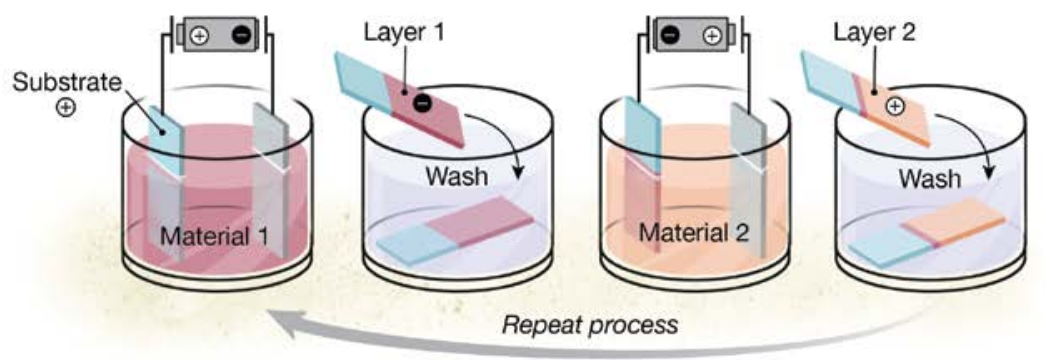

E Fluidic

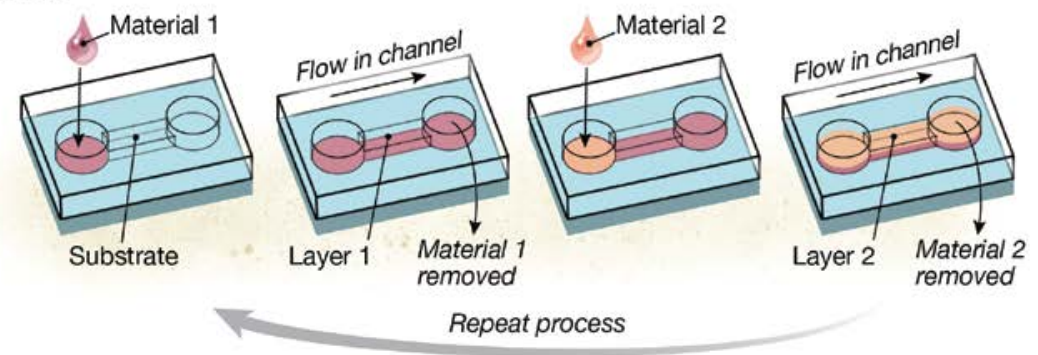


Fig. 3. Comparison between immersive and spin assembly. (A) Schematic comparison with different layer materials. (B) Comparison between an immersive-assembled film (left) and a spin-assembled film (right). Films are made of hydrophobically modified poly(ethylene oxide) and poly(acrylic acid). Adapted with permission from ref 44. Copyright 2008 American Chemical Society.

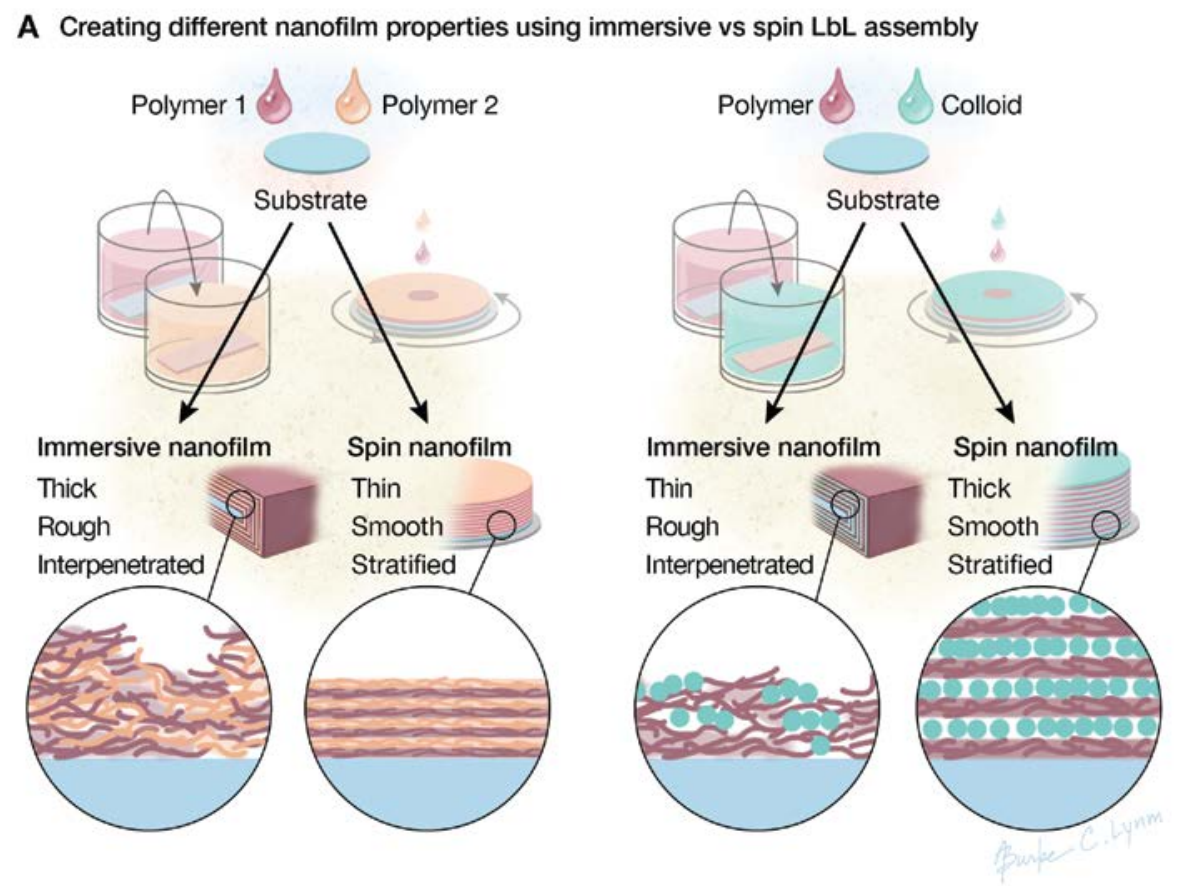

B Examples of polymer-polymer nanofilms using immersive vs spin LbL assembly
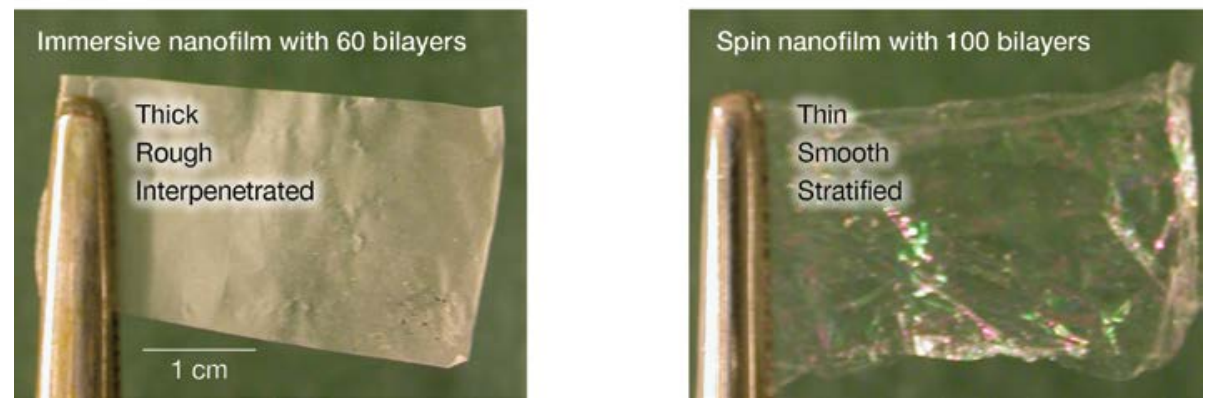
Table 1. Selected LbL assembly technologies and properties arising from using each technology. The table is intended to provide a general overview and is not exhaustive.

\begin{tabular}{|c|c|c|c|c|c|c|c|c|}
\hline \multirow[b]{2}{*}{$\begin{array}{l}\text { LbL ASSEMBLY } \\
\text { TECHNOLOGY }\end{array}$} & \multicolumn{2}{|c|}{ BUILDING BLOCKS } & \multirow{2}{*}{ Layer materials } & \multicolumn{2}{|c|}{ PROCESS PROPERUIES } & \multicolumn{3}{|c|}{ NANOFILMPROPERTIES } \\
\hline & Substrates & $\begin{array}{l}\text { Substrate } \\
\text { sizes }^{\star}\end{array}$ & & $\begin{array}{l}\text { Time per } \\
\text { layer }\end{array}$ & $\begin{array}{l}\text { Automated } \\
\text { (current status) }\end{array}$ & $\begin{array}{l}\text { Layer } \\
\text { thickness, } \mathrm{nm}^{\dagger}\end{array}$ & $\begin{array}{l}\text { Roughness, } \\
\text { nm }\end{array}$ & $\begin{array}{l}\text { Layer } \\
\text { structure }\end{array}$ \\
\hline Immersive & & $10 \mathrm{~nm}-1 \mathrm{~m}$ & & $10 s-12 h$ & & $<1-15$ & $1-20$ & Interpenetrated \\
\hline Dipping $(2,25)$ & Planar & $1-100 \mathrm{~mm}$ & Polymers, colloids & $\begin{array}{l}10-30 \mathrm{~s}(32) \text { or } \\
10-20 \mathrm{~min}^{\ddagger}(26)\end{array}$ & Yes & $1-2(25,26)$ & $1-10(44,49)$ & \\
\hline Dewetting (8) & Planar & $1-10 \mathrm{~mm}$ & Polymers, colloids§ & $30-60 \mathrm{~s}(8)$ & No & $1-2(8)$ & NR & \\
\hline Roll-to-roll (9) & Flexible planar & $\mathrm{r} 100 \mathrm{~mm}-1 \mathrm{~m}$ & Polymers & $2-5 \min (9,34)$ & Yes & $1-15(9,34)$ & $15-20(9,34)$ & \\
\hline Centrifugation $(5,6)$ & Particulate & $10 \mathrm{~nm}-10 \mu \mathrm{m}$ & Polymers, colloids & $20+\min \|(5,6)$ & No & $1-2(5,10)$ & $3-10(23,94)$ & \\
\hline $\begin{array}{l}\text { Calculated saturation } \\
(12,37)\end{array}$ & Particulate & $100 \mathrm{~nm}-1 \mu \mathrm{m}$ & Charged polymers & $5-10 \min (39)$ & No & $1-2(39)$ & NR & \\
\hline $\begin{array}{l}\text { Immersive immobilization } \\
\text { (33) }\end{array}$ & Particulate & $100 \mathrm{~nm}-1 \mu \mathrm{m}$ & Polymers & $40-50 \min (33)$ & Yes & $<1(33)$ & NR & \\
\hline Creaming (11) & Emulsion & $10 \mathrm{~nm}-1 \mu \mathrm{m}$ & Polymers, colloids & $0.5-12$ h $(11,36)$ & No & $1-7(36,38)$ & NR & \\
\hline Spin & & $1-100 \mathrm{~mm}$ & & $10 \mathrm{~s}-5 \mathrm{~min}$ & & $<1-2$ & $1-10$ & Stratified \\
\hline Spin $(14,42,43)$ & Planar & $1-100 \mathrm{~mm}$ & Polymers, colloids & $10-60 \mathrm{~s}(43)$ & Yes & $<1-2(42)$ & $1-10(44,46)$ & \\
\hline High gravity (15) & Planar & $1-10 \mathrm{~mm}$ & Polymers, colloids & $20 \mathrm{~s}-5 \min (15,49)$ & No & NR & $1-2(49)$ & \\
\hline Spray & & $10 \mathrm{~nm}-10 \mathrm{~m}$ & & $<1 s-24 h$ & & $<1-15$ & $1-10$ & Stratified \\
\hline Spray $(16,52)$ & Planar & $1 \mathrm{~mm}-10 \mathrm{~m}$ & Polymers & $<1-30 s(59)$ & Yes & $<1-5(16,51)$ & $1-10(51)$ & \\
\hline Atomization (17) & None & $10-100 \mathrm{~nm}$ & Charged polymers & $12-24 \mathrm{~h}(17)$ & No & $5-15(17)$ & NR & \\
\hline Spray immobilization (63) & Particulate & $10-100 \mathrm{~nm}$ & Polymers & $5-10 s(63)$ & Yes & $2-4(63)$ & NR & \\
\hline Electromagnetic & & $10 \mathrm{~nm}-100 \mathrm{~mm}$ & & $1 \mathrm{~s}-20 \mathrm{~min}$ & & $1-20,000$ & $10-30$ & Stratified \\
\hline $\begin{array}{l}\text { Electrodeposition }(18,20 \text {, } \\
67,73)\end{array}$ & Planar & $1-100 \mathrm{~mm}$ & Polymers, colloids & $1 \mathrm{~s}-20$ min 165,71$)$ & No & $\begin{array}{l}2-20,000 \text { ी } \\
(20,67,71)\end{array}$ & $10-30(66,70)$ & \\
\hline Magnetic $(19,76)$ & $\begin{array}{l}\text { Planar and } \\
\text { Particulate }\end{array}$ & $10 \mathrm{~nm}-100 \mathrm{~mm}$ & Polymers, colloids & $15-20 \min (19,76)$ & No & $1-2(19,75)$ & NR & \\
\hline Electro-immobilization (13) & Particulate & $10 \mathrm{~nm}-1 \mu \mathrm{m}$ & Charged polymers & $15-20 \min (13)$ & No & $2-3(13)$ & NR & \\
\hline Fluidic & & $100 \mathrm{~nm}-100 \mathrm{~mm}$ & & $10 \mathrm{~s}-45 \mathrm{~min}$ & & $<1-3$ & $1-11$ & NR \\
\hline क्र Microfluidic planar (22) & Planar & $10 \mu \mathrm{m}-100 \mathrm{~mm}$ & Polymers & $1-15 \min (80,81)$ & Yes & $<1-3(83,84)$ & $1-10(80,84)$ & \\
\hline $\begin{array}{l}\text { Microfluidic particulate } \\
(96-98)\end{array}$ & Particulate & $100 \mathrm{~nm}-10 \mu \mathrm{m}$ & Polymers & $10-60$ s (96-98) & Yes & $1-3(96,97)$ & NR & \\
\hline Fluidized bed (23) & Particulate & $1-10 \mu \mathrm{m}$ & Polymers & 3-5 $\min (23)$ & No & $2-3(23)$ & $9-11(23)$ & \\
\hline $\begin{array}{l}\text { Fluidic immobilization }(86, \\
87)\end{array}$ & Particulate & $100 \mathrm{~nm}-1 \mu \mathrm{m}$ & Polymers, colloids & $5-45 \min (86,87)$ & No & $1-2(87)$ & NR & \\
\hline Vacuum/filtration (21) & $\begin{array}{l}\text { Particulate } \\
\text { and fragile" }\end{array}$ & $100 \mathrm{~nm}-1 \mu \mathrm{m}$ & Polymers & $10-20 \min (95)$ & Yes & $1-2(94)$ & $5-10(94)$ & \\
\hline
\end{tabular}

NR indicates not reported in selected references.

"Typical order of magnitude substrate sizes indicated. Larger or smaller substrate sizes are possible.

t Typical thicknesses per layer for linearly growing films are indicated. Per layer thicknesses for exponentially growing films vary widely given the non-linear growth profile.

¥ Time with and without agitation, respectively.

§ Dewetting can utilize materials that are usually difficult to layer, e.g. materials with low charge or with low surface contact.

$\|$ Centrifugation processing time is highly variable due to manual pipetting and resuspension steps.

१ी Thickness is dependent on time.

** Fragile substrates such as mammalian cells can be layered using fluidic filtration 


\section{University Library}

\section{- M M I E E R VA A gateway to Melbourne's research publications}

Minerva Access is the Institutional Repository of The University of Melbourne

Author/s:

Richardson, JJ;Bjoernmalm, M;Caruso, F

Title:

Technology-driven layer-by-layer assembly of nanofilms

Date:

2015-04-24

Citation:

Richardson, J. J., Bjoernmalm, M. \& Caruso, F. (2015). Technology-driven layer-by-layer assembly of nanofilms. SCIENCE, 348 (6233), https://doi.org/10.1126/science.aaa2491.

Persistent Link:

http://hdl.handle.net/11343/90861 